\title{
Fetal Congenital Hepatic Cyst
}

\author{
Juan Piazze ${ }^{1 *}$ and Silvio Rea ${ }^{2}$ \\ ${ }^{1}$ ASL Frosinone (Casa della salute di Ceprano-Ospedale SS Trinità di Sora), Frosinone, Lazio, Italy \\ ${ }^{2}$ Chirurgia Oncologica, Università degli Studi de L'Aquila, Roma Italy
}

*Corresponding author: Juan Piazze, ASL Frosinone (Casa della salute di Ceprano-Ospedale SS Trinità di Sora), Frosinone, Lazio, Italy.

Received Date: May 06, 2020

Published Date: May 22, 2020

Keywords: congenital hepatic cyst, fetal liver ultrasound.

\section{Introduction}

Congenital hepatic cyst is a rare and nonsymptomatic condition in infants. Its incidence is $2.5 \%$ in the postnatal life with a much lower incidence in the prenatal period. Incidental finding on antenatal imaging is the most common presentation.

We present a case of a female new-born in which fetal ultrasound detected a cyst within the fetal liver. Postnatal imaging revealed a liver cyst in the right lobe of the liver, fortunately the neonate presented with no other intrahepatic structure affected. Liver function tests were normal and patient was asymptomatic after birth and in further controls. An abdominal ultrasound scan showed a complete disappearance of the cyst by 9 months.

\section{Case Presentation}

A 24-year-old female presented to our institution for her prenatal care at her first pregnancy.

At 22 weeks of gestation, a structural fetal sonogram did not report any substantial mass or abdominal disorder (gall bladder was described as visualized).

At $33+1$ weeks of gestation, a third trimester normal pregnancy evaluation was performed. The exam showed an echogenic structure in the upper right fetal abdomen, showing a dimension of $25 \times 27 \times 39 \mathrm{~mm}$ (Figure 1). No signs of ascitis or any other abdominal organ compromise were noted, including a normal fetal maternal Doppler velocimetry evaluation in a normal growth fetus. The gallbladder appeared unremarkable, with no intrahepatic biliary duct dilatation. A magnetic resonance imaging (MRI) was performed a week after, which confirmed the ultrasound scan diagnosis of congenital hepatic cyst.

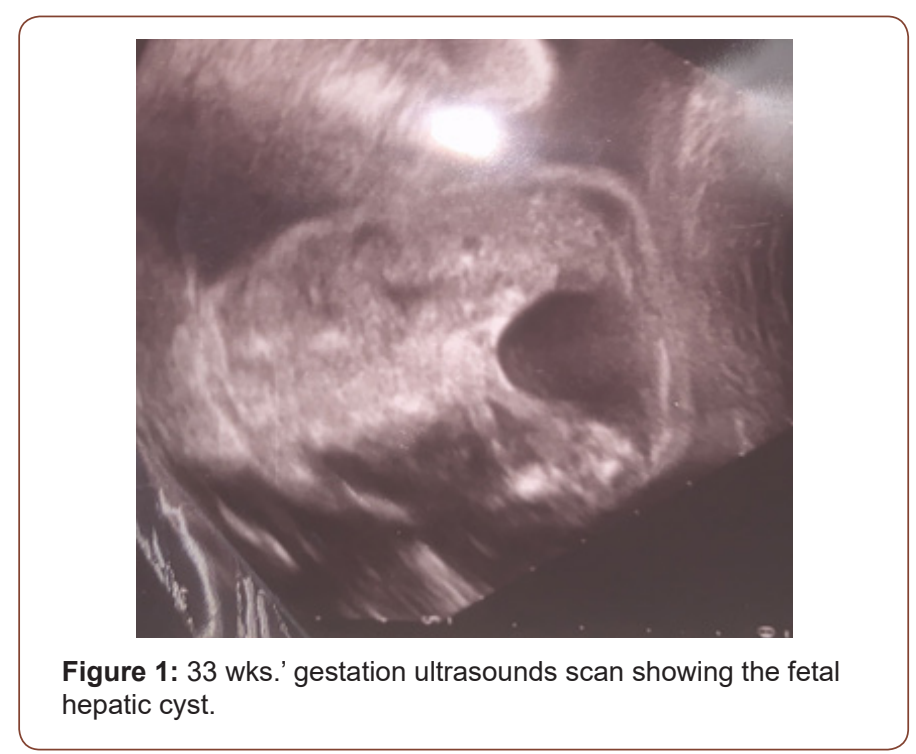

The female neonate was uneventful at birth ( 39 wks. gestation), with an optimal Apgar score and with a birth weight of $3220 \mathrm{~g}$. Neonatologist abdominal examination revealed a no distended 
abdomen, with no masses palpated. On the first day after birth, an abdominal sonogram revealed a hepatic cyst in the right lobe of the liver, measuring $30 \times 40 \mathrm{~mm}$. The infant remained asymptomatic and was discharged presenting a hepatic function panel and total bilirubin within normal ranges. The baby was evaluated by the paediatric gastroenterologist who recommended repeating the sonogram to ensure stability. As such, a follow-up sonogram was performed at 6 months of life showing a cyst measuring $21 \times 22.5 \mathrm{~cm}$ in the right lobe of the liver with no increased vascularity. She was re-evaluated by paediatric gastroenterologist at 9 months of age when a new ultrasound was performed, showing the complete disappearance of the simple hepatic cyst.

\section{Comment}

The incidence of congenital hepatic cyst in the postnatal life is $2.5 \%$. They are more common in girls, not associated with cysts in other organs, and rarely communicate with the biliary tree [1].

Incidental finding of an asymptomatic lesion on antenatal imaging is the most common presentation of a congenital hepatic cyst. Cholestasis may also be observed in some cases due to the compression of the hepatic parenchyma and the biliary system by a large cyst. Ultrasonography shows the congenital hepatic cyst as an anechoic unilocular fluid-filled space with a posterior acoustic enhancement. Magnetic resonance imaging (MRI) typically reveals a well-demarcated water-attenuated lesion without enhancement after gadolinium with low-intensity signal on $\mathrm{T} 1$ and high-intensity signal on T2 images. In our case, MRI was indicated, confirming the fetal US scan findings. Management of congenital hepatic cyst is conservative with periodic ultrasound monitoring to ensure their stability. Regarding surgical interventions, only few infants with simple hepatic cysts required surgical intervention due to gradual enlargement and symptoms, and the others needed no further intervention [2,3]. A simple US scan revealed the fetal hepatic cyst at 9 months extra uterine life in this case.

\section{Final Remarks}

Congenital hepatic cyst is a condition with a narrow differential diagnosis. Surgical treatment with partial or total removal of the cyst is the favoured treatment in neonates with a large symptomatic lesion. Ultrasound surveillance is important in order to estimate an adequate follow-up and the eventual decision for a surgical intervention.

\section{Acknowledgement}

None.

\section{Conflict of Interest}

Authors declare no conflict of interest.

\section{References}

1. Viswanathan S, Kumar D (2014) Diagnostic challenge of large congenital liver cyst in the newborn. Pediatr Int 56(2): 267-270.

2. Bruns NE, Asfaw SH, Stackhouse KA, Falk GA, Magnuson DK, et al. (2015) Laparoscopic excision of a ciliated hepatic foregut cyst in a child: a case report and review of the literature. Ann Med Surg (Lond) 4(4): 467-469.

3. Rogers TN, Woodley H, Ramsden W, Wyatt JI, Stringer D (2007) Solitary liver cysts in children: not always so simple. J Pediatr Surg 42: 333-339. 